\title{
APPLICATION OF FINSLERIAN SPACES IN ELECTROMAGNETISM AND SOLID MECHANICS
}

POOJA SWAROOP SAXENA

DIT University, Dehradun, Uttarakhand, India

\begin{abstract}
In Finsler geometry, each point of a base manifold can be endowed with coordinates describing its position as well as a set of one or more vectors describing directions. Finsler geometry encompasses Riemannian, Euclidean, and Minkowskian geometries as special cases, and thus it affords great generality for describing a number of phenomena in physics. This paper is involving application of Finsler geometry in Electromagnetism and continuum mechanics of solids is reviewed. With the help of finsler Gauge transformation it has been shown the geometrization of Electromagnetism in Finsler spaces.

KEYWORDS: Finslerian Spaces
\end{abstract}

Received: May 06, 2020; Accepted: May 26, 2020; Published: Jun 30, 2020; Paper Id.: IJMPERDJUN2020186

\section{INTRODUCTION}

The most known geometrical approaches to unification are the theories of Weyl and Kaluza-Klein, which aim to geometrize electromagnetism like gravitation. These theories faced with series problems such as, in Weyl's theory, the norms of vectors are not invariant under parallel transport, and in the approach of Kaluza-Klein theories, electrodynamics is geometrized in a five dimensional space-time. Finsler geometry is an alternative approach to geometrization of fields, and its fundamental idea can be traced back to a lecture of Riemann, in 1854 [2].In this lecture Riemann suggested that the positive fourth root of a fourth order differential form might serve as a metric function. This function has three properties that it is convex and common with the Riemannian quadratic form itis positive and homogeneous of degree one in the differentials. Therefore, it is anatural generalization of the notion of distance between two neighboring points $x i ; x i+d x i$ to consider as given by some function $F(x i$; $d x i)$, where $i=$ $0 \ldots . . n$, satisfying these three properties. Finsler geometry was first applied in gravitational theory, and this application lead to corrections to observational results predicted by general relativity $[4,6,13,20]$.

As mentioned before, the main application of Finsler geometry is the geometrization of electromagnetism and gravitation. A Finslerian approach to this geometrization was first introduced by Randers [3], but in his work Finsler geometry was not mentioned, although it was used. Randers metric produces a geodesic equation identical with Lorentz equation for a charged particle. But themetric depends on $q / m$ and defines a different space for each type of particle [21].

In the approach given in this study, unified theory of gravitation and electrodynamics is developed from a Finslerian tangent space gauge transformation [14,15].The transformation physically interpreted as containing physical fields and the resulting metric is similar to the metric introduced by Kaluza-Klein, but has different physical interpretations in the scheme of Finsler geometry $[16,17,18,19]$ 
Mechanical behavior of homogeneous isotropic elastic solids can be described by constitutive models that depend only on local deformation, for example, some metric or strain tensor that may generally vary with position in a body. Materials with microstructure require more elaborate constitutive models, for example, describing lattice orientation in anisotropic crystals, dislocation mechanisms in elastic-plastic crystals, or cracks or voids in damaged brittle or ductile solids. In conventional continuum mechanics approaches, such models typically assign one or more time- and position dependent vector(s) or higher-order tensor(s), in addition to total deformation or strain, that describe physical mechanisms associated with evolving internal structure.

\section{FINSLER GAUGE TRANSFORMATIONS}

If a particle in a space-time moves along a curved, non-geodesic path, then it is said that the particle is under the influence of some external force. In such a case, an external force term is added to the equation of motions to explain the path of motion. Alternative point of view is that motion can be explained by a new metric, which would result from a gauge transformation. In this way, physical force fields can be geometrized, and general relativistic idea of space time curvature determining the path of the particle will also include fields other than gravitation. For this purpose a class of gauge transformations which act on tangent space is considered [14]

Under these kind of transformations, the tangent vector $y$, transforms as

$$
\bar{y}^{\mu}=Y_{a}^{* \mu} y^{\nu}
$$

Where $\mu_{y} v_{y} \ldots=0,1,2,3$ are indices corresponding the space components, and

$$
Y_{v}^{* \mu}=\frac{\partial y^{\mu}}{\partial y^{2}}
$$

$$
Y_{\alpha}^{* \mu} Y_{v}^{\alpha}=\delta_{v i}^{\mu}
$$

Where, $Y_{v}^{\mu}=\frac{\partial y^{\mu}}{\partial y^{v}}$ is the inverse transformation, and these transformations $\left(Y_{v}^{\mu}\right)$ are called $Y$ transformations. Even though the transformation does not act on the base space coordinates, it will seen to produce changes in the base space. Thus, these transformations also depend on the base coordinates, such as

$$
Y_{q u}^{e \mu}=Y_{q}^{e \mu}(x, y)
$$

The $Y$ transformation of the metric tensor is given as

$$
\bar{g}_{\mu v}(x, y)=Y_{\mu k}^{\alpha}(x, y) Y_{\nu}^{\beta} g_{\alpha \beta}(x, y)
$$

Under this transformation, Finsler metric function is invariant, such as

$$
\begin{aligned}
\bar{F}^{2}(x, \bar{y}) & =\bar{g}_{\mu v} \bar{y}^{\mu} \bar{y}^{v}=g_{\sigma \beta}(x, y) Y_{\mu}^{\alpha} Y_{v}^{\beta} Y_{\gamma}^{\star \mu} Y_{\sigma}^{v} y^{\gamma} y^{\sigma} \\
& =F^{2}(x, y)
\end{aligned}
$$


Here $y^{v}$ is the contra variant vector and the covariant vector associated with it is $y_{\mu \nu}$, where $y_{\mu}=g_{\mu \nu} y^{\nu}$. Covariant vector $y_{\mu \text { transforms as }}$

$$
\bar{y}_{\mu}=Y_{\mu}^{a} y_{\alpha}
$$

Since

$$
\frac{\partial \bar{y}_{\mu}}{\partial \bar{y}^{v}}=\bar{g}_{\mu v} \quad=Y_{\mu}^{\alpha} Y_{v}^{\beta} g_{\alpha \beta}+Y_{v}^{\beta} \frac{\partial Y_{\mu}^{\alpha}}{\partial y^{\beta}} y_{\alpha}
$$

The $Y$ transformation of the Finslerian metric tensor does not yield a tensor unless Type equation here.

$$
\frac{\partial Y_{\mu}^{\alpha}}{\partial y^{\beta}} y_{\alpha}=0
$$

The condition (3.9) is called as the metric condition [5]. It is obvious that $Y$ transformations, when $Y_{v}^{\mu}$ is a function of $x$ only, that is

$$
Y_{v}^{\mu}=Y_{v}^{\mu}(x)
$$

Satisfy the metric condition. These type of transformations are called K-group or linear transformations [5]. $Y$ transformations can be interpreted as the transformations from an original space where there exists no external field, to a space that also contains external fields which are turned on by some physical potentials contained in $Y_{v}^{\mu}[16]$.

A specfic example to $Y$ transformations was given as [14],

$$
Y_{v}^{\mu}=\delta_{v}^{\mu}-B^{-2}\left[1-\left(1+k B^{2}\right)^{1 / 2}\right) B_{v}^{\mu}
$$

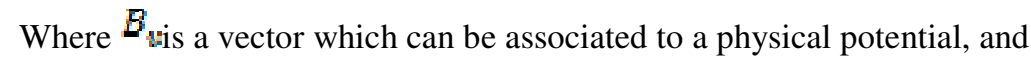

$$
B^{2}=g_{w} B^{H} B^{v}
$$

Here $k$ is a constant depending on the physical space that will be geometrized. The inverse transformation is given by the inverse of the matrix (2.11), such as Type equation here.

$$
Y_{\mu}^{* v}=\delta_{\mu}^{v}-B^{-2}\left\{1-\left(1+k B^{2}\right)^{-1 / 2}\right\} B^{v} B_{\mu}
$$

\section{APPLICATIONS IN SOLID MECHANICS}

The first application of Finsler geometry to finite deformation continuum mechanics is credited to Ikeda [23], who developed a director theory in the context of (pseudo-) Finslerian manifolds. A slightly earlier work [24] considered a generalized space (not necessarily Finslerian) comprised of finitely deforming physical and geometrical fields. Paper [23] is focused on kinematics and geometry: descriptions of deformations of the continuum and the director vector felds and their possible interactions, metric tensors (i.e., fundamental tensors), and gradients of motions. Covariant differentials are defined that can be used in field theories of Finsler space [25, 30]. Essential concepts from [23] are reviewed and analyzed next. 


\section{KINEMATICS AND GAUGE THEORY ON A FIBER BUNDLE}

The second known application of Finsler geometry towards finite deformation of solid bodies appears in Chapter 8 of the book of Bejancu [1]. Content in [1] extends and formalizes the description of Ikeda [23] using concepts of tensor calculus onthe fiber bundle of a (generalized pseudo-) Finsler manifold. Geometric quantities appropriate for use in gauge-invariant Lagrangian functions are derived. Relevant features of the theory in [1] are reviewed and analyzed in what follows next. Define $\zeta=(Z, \pi, M, U)$ as a fiber bundle of total space $\mathrm{Z}$, where $\pi: Z \rightarrow M$ is the projection to base manifold Mand $\mathrm{U}$ is the fiber. Dimensions of $M$ and $U$ are $n(n=3$ for a solid volume $)$ and $p$, respectively; the dimension of $Z$ is $r=n+p$. Coordinates on $\mathrm{Z}$ are $\left\{X^{A}, D^{\alpha}\right\}$ where $\mathrm{X} \in \mathrm{M}$ is a point on the base body in its reference configuration, and $\mathrm{D}$ is a director of dimension $\mathrm{p}$ that essentially replaces multiple directors of dimension 3 .The natural basis on is the field of frames

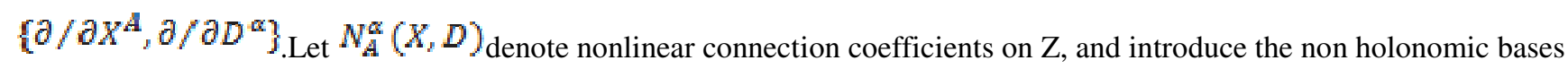
$\frac{\delta}{\delta X^{A}}=\frac{\partial}{\partial X^{A}}-N_{A}^{\alpha} \frac{\partial}{\partial D^{\alpha^{\gamma}}} \quad \partial D^{\alpha}=d D^{\alpha}+N_{A}^{\alpha} d X^{A}$ Unlike $\left\{\partial / \partial X^{A}\right\}, t$ hese nonholonomic bases obey simple transformation laws; $\left\{\delta / \delta X^{A}, \partial / \partial D^{\alpha}\right\}$ serves as a convenient local basis for TZ adapted to decomposition into horizontal and vertical distributions:

$$
T Z=H Z \oplus V Z
$$

\section{TOWARDS A NEW THEORY OF STRUCTURED MEDIA}

Finsler geometry can be applied to describe physical problems in deformable continua with evolving microstructures in a manner somewhat analogous to the phase field method. Phase field theory [8] encompasses various diffuse interface models wherein the boundary between two (or more) phases or states of material is distinguished by the gradient of a scalar field called an order parameter. The order parameter, denoted herein $\eta_{\text {by typically varies continuously between values of }}$ zero and unity in phases one and two, with intermediate values in phase boundaries. Mathematically,

$$
\begin{aligned}
& \eta(X)=0 \forall \text { Xe phase } 1 \\
& \eta(X)=1 \forall \text { Xe phase } 2 \\
& \eta(X)=(0,1) \forall \text { Xe interface, }
\end{aligned}
$$

Physically, phases might correspond to liquid and solid in melting-solidification problems, austenite and martensitein structure transformations, vacuum and intact solid in fracture mechanics, or twin and parent crystal in twinning descriptions. Similarities between phase field theory and gradient-type theories of continuum mechanics are described in [7]; both classes of theory benefit from regularization associated with a length scale dependence of solutions that can render numerical solutions to governing equations mesh independent. Recent phase field theories incorporating finite deformation kinematics include [28] for martensitic transformations, for fracture, [10] for amorphization, and [11] for twinning..Deformation twinning involves shearing and lattice rotation/reflection induced by mechanical stress in a solid crystal. The usual elastic driving force is a resolved shear stress on the habit plane, in the direction of twinning shear. 
Twinning can be reversible or irreversible depending on material and loading protocol; the physics of deformation twinning is described more fully in [28] and Chapter 8 of [12].

\section{REFERENCES}

1. A Bejancu. Finsler Geometry and Applications, Ellis Horwood, New York, NY, USA, 1990.

2. G. F. B. Riemann, 'Über die Hyphothesenwelche der Geometriezu Grunde liegen', Habilitation thesis, University of Göttingen, (1854).

3. G.Randers, Phys.Rev.59 (1941), 195

4. G. S. Asanov, IlNuovoCimento, 49(1979), 221.

5. G.S.Asanov, 'Finsler Geometry, Relativity and Gauge Theories', D. Reidel, Dordrecht, (1985)

6. G. Yu. Bogolovsky, Il NuovoCimento, $40 B$ (1977), 99.

7. G. Z. Voyiadjis and N. Mozaffai, "Nonlocal damage model using the phase field method: theory and applications," International Journal of Solid and Structures, Vol. 50, No. 20-21 pp 3136-3151, 2013

8. H. Emmerich, The Diffuse Interface Approach in Material Science: Thermodynamic concepts and Applications of Phase Field Models, Springer, Berlin, Germany, 2003

9. I W. Roxburgh, Gen. Rel. Grav. 24 (1992), 419.

10. J. D. Clayton, "Phase field theory and analysis of pressure- shear induced amorphization and failure in boron carbide ceramic," AIMS Materials Science, Vol. 1, No.3, pp. 143-158, 2014

11. J. D. Clayton and J. Knap, "A phase field model of deformation twinning: nonlinear theory and numerical simulations,"Physica D. Nonlinear Phenomenon, Vol. 240, No. 9-10, pp. 841-858, 2011

12. J. D. Clayton, Nonlinear Mechanics of Crystal, Springer, Dordrecht, The Netherlands, 2011

13. M. Matsumato, Rep. Math. Phys. 8(1975), 103

14. R.G. Beil, Internat, J.Theoret. Phys. 31, No: 31, (1992), 1025

15. R.G. Beil, Internat, J. Theoret. Phys, 36, No: 2, (1987), 189

16. R. G. Beil, Contem. Math. 196 (1996), 265

17. R. G. Beil, Contemp. Math. 196 (1996), 261

18. R.G.Beil, Internat. J. Theoret. Phys. 32, No: 6, (1993), 1021

19. R.G. Beil, Internat. J. Theoret. Phys. 28, No: 6, (1989), 659

20. R. K. Takavol, N. Van der Bergh, Gen. Rel. Grav. 18 (1986), 849.

21. R.S. Ingarden, Tensor, N.S.30 (1976), 201

22. S. F. Rutz, Gen. Rel. Grav. 25, No:11, (1993), 1139

23. S. Ikeda, "Aphysico-geometrical consideration on the theory of directors in the continuum mechanics of oriented media," The Tensor society, Tensor: New series, Vol27, pp361-368, 1973

24. S. Ikeda, "A geometrical construction of the physical interaction field and its application to the rheological deformation field," Tensor, Vol. 24, pp 60-68, 1972 
25. S. Ikeda, "On the theory of fields in Finsler space.," "Journal of Mathematical Physics, Vol 22 No. 6, pp 1215-1218, 1981

26. T. Ishikawa, J. Math. Phys. 22(1981), 995.

27. V. I. Levitas, V. A. Levin, K.M. Zingerman and E. I. Freiman, “Displacive phase transitionsat large strains: phase field theory and simulations, "Physical review Letters, Vol. 103, No. 2 , Article ID 025702,2009

28. V. S. Boiko, R. I. Garber and A.M. Kosevich, Reversible Crystal Plasticity, AIP Press, New York, NY, USA, 1994

29. Y. Tokano, Lett. Nuovo Cimento 10(1974), 307.

30. Y. Takano, "Theory of fields in Finsler space I, Progress of Theoretical Physics, Vol. 40, pp 1159-1180, 1968

31. Saxena, Pooja S. "The Concircular Vector Fields." International Journal of Mathematics and Computer Applications Research (IJMCAR) 9.1 (2019):7-10

32. Rastogi, S. C. "On Some New Tensors and Their Properties in a Five-Dimensional Finsler Space-III." IMPACT: International Journal of Research in Applied, Natural and Social Sciences (IMPACT: IJRANSS) 7.9 (2019):1-14

33. Rastogi, S. C. "D-Concurrent Vector Field in a Finsler Space of Three-Dimensions." IMPACT: International Journal of Research in Applied, Natural and Social Sciences (IMPACT: IJRANSS) 7.11 (2019):15-28

34. Rastogi, S. C. "Study of certain new tensors in a Finsler space of three-dimensions." IMPACT: International Journal of Research in Applied, Natural and Social Sciences (IMPACT: IJRANSS) 7.11 (2019): 1-14.

35. Mahajan, Anmol, and Pratham Chhabra. "Design and Static Analysis of Electromagnetic Shock Absorber Using Electromagnetic Induction." International Journal of Mechanical and Production Engineering Research and Development (IJMPERD) $5.6(2015): 59-64$ 